Ann. Biol. anim., Bioch., Biophys., I966, 6 (2), 255-259.

\title{
NOTE SUR LA COMPOSITION EN ACIDES AMINÉS DU CONTENU DE CAECUM DE RATS AXÉNIQUES ET DE RATS TÉMOINS ( $\left.{ }^{1}\right)$
}

\author{
litiennette COMBE* et R. PION \\ Laboratoire d'Études des. Lítabolismss, \\ Centre national de Recherches zootechniques, 78-Jouy-en-Josas
}

\section{INTRODUCTION}

I'hypertrophie du cæecum est l'une des caractéristiques les plus frappantes des rats axéniques.

I, a paroi de tels cæcums, en effet, pèse deux à trois fois plus et renferme cinq à six fois plus de contenu frais (GORDON, 1959), et deux fois et demie à quatre fois plus d'azote (Combe et al., Ig66) que celle des témoins ( ${ }^{1}$ ).

Le fractionnement du contenu de cæcum, soit par l'éthanol 80 p. Ioo et par l'acide trichloracétique Io p. Ioo (CомBE et al., I966), soit par une solution saline à 9 p. I 000 (LINDSTEDT et al., I 965) a montré que ces différences portent surtout sur les composés solubles.

Toutefois, l'ammoniaque, l'urée et les acides libres présents dans la fraction éthanolique ne rendent compte que de I3 à $26 \mathrm{p}$. Ioo de l'azote présent dans cette fraction et nous avons été amenés à étudier la composition en acides aminés des composés azotés de poids moléculaire plus élevé présents dans les différentes fractions.

\section{MATÉRIEL E'T MÉTHODES}

Des lots de 5 à 10 rats (p. $m .=260$ g) axéniques, conventionnels, traditionnels reçoivent un régime stérilisé à 22 p. Ioo de matières azotées.

Les prélèvements des contenus de cæcum et l'extraction des composés solubles dans l'éthanol 80 p. roo ont été faits comme nous l'avons décrit précédemment (CoMBE et al. ig66). Le résidu de l'extraction ethanolique a été traité ensuite avec de l'acide trichloracétique ıo p. Ioo (ATC).

(1) Nous avons utilisé deux catéurories de rats ténoins; des rats Lobund dits "conventionnels ", issus de rats nés et sevrés à l'état axénique, et vivant ensuite dans un milieu non aseptique ; des rats " traditionmels", de souche Wistar, obtenus dans la riterie du laboratoire.

* Adresse actuelle : Station d'Étude des Métabolismes, C.R.Z.V., Theix, Saint - Cienès - Champanelle, 63 Puy - de - Dôme. 
Nous avons ainsi obtenu pour chacun des lots expérimentaux trois fractions contenant :

- les composés solubles dans l'éthanol 80 p. Ioo: fraction I,

- les composés solubles dans l'ATC io p. roo: fraction II,

- les composés insolubles à la fois dans l'éthanol et l'ATC : fraction III.

Dans chaque fraction, nous avons déterminé l'azote (Kjeldahl) et les acides aminés par chromatographie quantitative sur colonne selon MOORE et STEIN (1958) après hydrolyse HCl 6 N à reflux.

\section{RÉSUI,TATS}

La répartition de l'azote entre les trois fractions chez les rats axéniques est très différente de celle que l'on trouve chez les témoins:

TABLEAU I

Répartition centésimale de l'azote

du contenu de cacum des rats entre différentes fractions

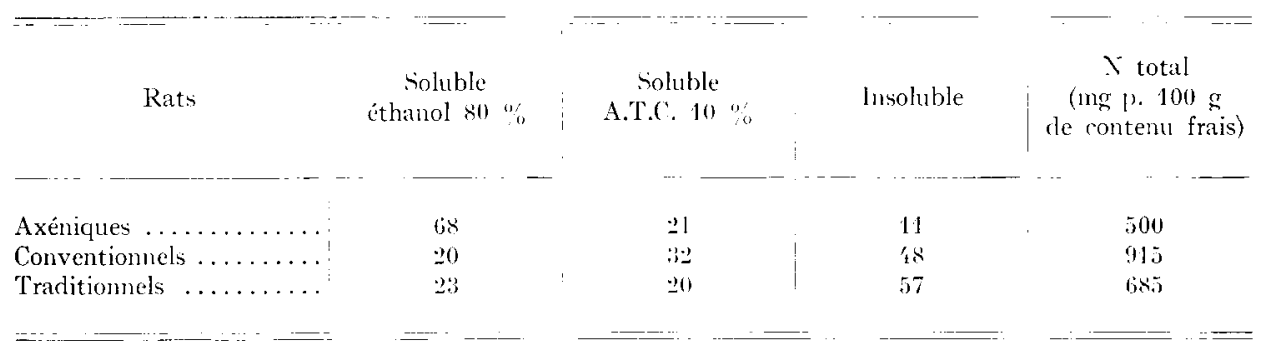

Les teneurs en acides aminés, déterminées après hydrolyse acide, dans les diverses fractions, sont données dans le tableau 2. Notons que les acides aminés et 1'ammoniac ( $\left.{ }^{1}\right)$ retrouvés dans les fractions II et III rendent compte respectivement de 8 I, 5 et 98 p. Ioo de 1'azote total trouvé dans ces fractions.

Ces mêmes composés, dosés après hydrolyse acide dans la fraction I (extrait éthanolique), représentent 65 à $72 \mathrm{p}$. Ioo de l'azote total de cette fraction (du fait de la présence d'amides, de sucres aminés, d'ammoniac et d'urée dans cette fraction.)

Les trois fractions du contenu de cæcum des rats axéniques ont des compositions en acides aminés combinés bien particulières :

- La composition en acides aminés de la fraction III du contenu de crecum des rats axéniques est assez voisine de celle de la paroi intestinale. Elle en diffère toutefois notamment par sa faible teneur en lysine et sa teneur élevée en sérine et en tyrosine. La faible proportion de lysine pourrait s'expliquer par l'action préférentielle protéolytique de la trypsine qui n'a pas encore disparu du contenu digestif au niveau du cæcum des rats axéniques, puisque BorGSTrom et al. (x959) en ont trouvé dans les fèces. I a présence de telles enzymes assez riches en thréonine et sérine pourraient également expliquer 1'importance de ces acides aminés dans le contenu de cæcum des rats axéniques. 
- I a fraction II, soluble dans l'ATC Io p. Ioo, a une composition en acides aminés bien différente de celle des autres fractions étudiées. Elle se rapprocherait de la composition en acides aminés des glycopeptides analysés par AlaIs et Jolikis

\section{TABLEAU 2}

Composition en acides aminés ( $\mathrm{g} / \mathrm{1} 6 \mathrm{~g} \mathrm{~N})$ de différentes fractions

du contenu de cecum chez des rats axéniques

et des rats témoins (conventionnels et traditionnels)

\begin{tabular}{|c|c|c|c|c|c|c|c|c|c|}
\hline \multirow[b]{2}{*}{ Rats $\left\{\begin{array}{l}\text { Nombre......... } \\
\text { Lots........... }\end{array}\right.$} & \multicolumn{2}{|c|}{ Fraction I } & \multicolumn{3}{|c|}{ Fraction II } & \multicolumn{3}{|c|}{ Fraction III } & \multirow{2}{*}{$\begin{array}{c}\text { Paroi } \\
\text { intestinale } \\
\text { de rats } \\
\text { trad. lavée } \\
\text { au T.C.A. }\end{array}$} \\
\hline & $\begin{array}{c}8 \\
\text { Axén. }\end{array}$ & $\begin{array}{c}10 \\
\text { Axén. }\end{array}$ & $\begin{array}{c}9 \\
\text { Axén. }\end{array}$ & $\begin{array}{c}8 \\
\text { Conv. }\end{array}$ & $\begin{array}{c}5 \\
\text { Trad. }\end{array}$ & $\begin{array}{c}9 \\
\text { Axén. }\end{array}$ & $\begin{array}{c}8 \\
\text { Conv. }\end{array}$ & $\stackrel{5}{\text { Trad. }}$ & \\
\hline Ac. aspartique ......... & 11,4 & 10,25 & 11,6 & 8,6 & 9,2 & 7,8 & 8,4 & 9,2 & 8,8 \\
\hline Thréonine $\ldots \ldots \ldots \ldots$ & 3,4 & 3,15 & $6, t$ & 3,2 & 3,3 & 5,2 & 4,35 & 4,7 & 4,6 \\
\hline Sérine $\ldots \ldots \ldots \ldots \ldots$ & 5,2 & 4,8 & 9,05 & 11,45 & 11,7 & 6,5 & 5,2 & 5,9 & 4,7 \\
\hline Ac. glutamique $\ldots . .$. & 17,0 & 14,6 & 17,2 & 23,4 & 23,5 & 15,7 & 15,7 & 15,4 & 12,6 \\
\hline Proline $\ldots \ldots \ldots \ldots \ldots \ldots$ & 5,65 & 5,2 & 4,7 & 3,9 & 4,1 & 5,6 & 3,9 & 3,6 & 4,8 \\
\hline Glycine . . . . . . . . . . & 3,35 & 3,15 & 3,2 & 2,7 & 2,8 & 1,9 & 4,2 & 3,1 & 5,65 \\
\hline Alanine $\ldots \ldots \ldots \ldots \ldots$ & 2,9 & 2,6 & 2,9 & 2,6 & 2,6 & 4,7 & $5,-1$ & 5,6 & 5,45 \\
\hline Valine .............. & 3,9 & 3,6 & 4,0 &, \pm 7 & 4,9 & $6,-4$ & 5,8 & 5,8 & 5,8 \\
\hline Méthionine $\ldots \ldots \ldots \ldots$ & 1,8 & $1, !$ & 0,6 & $1,: 3$ & 1,1 & 2,5 & 2,9 & 2,3 & 2,3 \\
\hline Isoleucine $\ldots \ldots \ldots \ldots$ : & 3,3 & 3,0 & 3,7 & 5,9 & 6,1 & 4,6 & 4,9 & 4 & 4,8 \\
\hline Leucine $\ldots . . . . . .$. & 3,8 & 3,65 & 3,3 & 2,5 & 2,4 & 8,0 & 7,3 & 8,1 & 8,25 \\
\hline Tyrosine ............ & 2,6 & 3,45 & 1,4 & 0,4 & 0,5 & 5,0 & 3,5 & 4,3 & 3,6 \\
\hline Phénylalanine ...... & 2,45 & 2,0 & $*$ & 1,2 & 0,9 & 4,15 & 1,4 & 4,9 & $\therefore, 7$ \\
\hline Lysine . . . . . . . . . . & 3,55 & 3,05 & 5,35 & 5,9 & 5,9 & 3,7 & 4,7 & 5,45 & 8 \\
\hline Histidine... & 1,35 & 1,35 & 1,75 & 1,55 & 1,5 & 2,0 & 1,9 & 2,05 & 2,3 \\
\hline Arginine . . & 2,74 & 2,6 & 2,8 & 1,7 & 1,0 & 5,1 & 1,6 & 4,7 & 6,6 \\
\hline Rendement azoté... & 65 & 72 & 71,3 & 80 & 82 & 90 & 85 & 86 & \\
\hline
\end{tabular}

* N’a pu être dosé par suite de la présence de sucres aminés.

(I964). Très riche en acides aminés hydroxylés (sérine et thréonine) et en glutamine (ou en acide glutamique) et lysine, la fraction II est pauvre en glycine, leucine et tyrosine. Ces particularités pourraient s'expliquer par la présence en quantités importantes de mucoprotéines, mises en évidence par LINDSTEDT et al. (I965) dans le contenu digestif de rats axéniques. En effet, d'après WERNER (I953), les mucoprotéines du tube digestif sont particulièrement riches en thréonine et pauvres en tyrosine, phénylalanine et méthionine.

- Dans la fraction I (extrait éthanolique du contenu de cæcum des rats axéniques) la composition en acides aminés combinés est voisine de celle des acides aminés libres (que nous avons précédemment analysés et dosés dans les mêmes échantillons (CombE et al., I g65). Seules les proportions plus élevées de quelques composés: de l'acide aspartique (ou de l'asparagine) et de l'acide glutamique (ou de la glutamine), entraînent une diminution passive de 20 à $30 \mathrm{p}$. Ioo de celles de la plupart des autres composés : thréonine, sérine, glycine alanine, isoleucine, leucine, phénylalanine en particulier. 
L'action de la flore sur la composition en acides aminés du contenu de cæcum est caractéristique pour chaque fraction :

- I)ans la fraction III, on observe une diminution importante de la teneur en proline et une augmentation importante de la teneur en lysine, peut être liées à la présence de corps microbiens. La teneur en glycine est également fortement diminuée, ainsi qu'à un moindre degré les teneurs en thréonine, sérine, valine, leucine, tyrosine, arginine. Ces diminutions sont compensées par des augmentations du même ordre des teneurs en acide aspartique (ou asparagine) alanine. phénylalanine.

Les caractéristiques de la composition en acides aminés de la fraction II sont elles aussi modifiées par la présence de la flore intestinale : la teneur en sérine est encore plus élevée que chez les rats axéniques, tandis que la teneur en thréonine est deux fois plus faible, et même inférieure à celle de la fraction III. I a glycine, l'alanine, la leucine, l'arginine et surtout la tyrosine sont en quantités encore plus faibles que chez l'animal axénique, tandis que la teneur en méthionine, quoique plus élevée que chez l'animal axénique, reste inférieure à celle de la fraction III. Les teneurs en acide glutamique (ou glutamine) et en isolencine deviennent très élevées. La teneur en acide aspartique (ou asparagine), plus faible que chez l'animal axénique, est comparable à celle de la fraction III.

Nous avions remarqué précédemment (Combe et al. Ig66) que la flore, tout en diminuant considérablement la quantité d'acides aminés libres (fraction I) avait également pour conséquence, une augmentation des teneurs en lysine, tyrosine et citrulline de cette fraction.

\section{CONCLUSION}

Ces résultats conduisent à poser le problème de l'origine de différents composés azotés présents dans le contenut de cæcum.

En ce qui concerne les composés solubles dans l'éthanol, on pourrait envisager l'origine endogène des acides aminés libres retrouvés en grande quantité dans le cæcum des rats axèniques, car ils n'ont pas l'occasion d'être métabolisés par la flore. Certains composés retrouvés en plus grande quantité dans le contenu de cacum des rats témoins abritant une flore intestinale très banale, seraient des produits du métabolisme bactérien tel l'ammoniaque et la citrulline.

En ce qui concerne les composés insolubles, l'analyse des résultats du tableau. 2 nous permet de faire quelques conclusions. - L,a fraction III des rats axéniques correspond à des desquamations de l'épithélium intestinal et la fraction III des rats témoins renferme des corps bactériens (riches en lysine). - Chez les axéniques, 1a fraction. II contient des mucoprotéines et en présence de la flore, on observe la disparition de la thréonine, de la tyrosine et de la méthionine (acides aminés caractéristiques des mucoprotéines selon WERNER). Mais la flore semble provoquer également une augmentation des teneurs en sérine et une diminution de la teneur en glycine. La diminution de la leucine permet de retrouver un rapport leucine/isoleucine comparable à celui des protéines animales.

Ces remarques mettent en lumière l'importance de la flore intestinale dans les phénomènes de digestion chez l'animal. 


\section{SUMMARY}

\section{NOTE ON THE AMINO ACID COMPOSITION OF THE CONTENTS OF THE CECUM} OF AXENIC AND CONTROL RATS

The contents of the cæcum of germ-free rats and conventional rats with the normal intestinal flora were compared by estimation of the amino acid composition. Substances soluble in 80 per cent ethanol (fraction I), those insoluble in ethanol but soluble in so per cent trichloroacetic acid (fraction II) and those insoluble in both ethanol and trichloroacetic acid (fraction III) were estimated.

The results summarized in table 2 show that fraction III of the germ-free rats correspond to the desquamation of the epithelium of the intestine, and that of the controls includes bacteria, rich in lysine.

In the germ-free rats fraction II contains mucoproteins, but in the presence of the flora the proteins analyzed contain little threonine (of which. with lysine, there is an abundance in the germfree rats) tyrosine and methionine. Similarly the flora seems to increase the contents of serine and decrease the glycine content. Because of the reduction in leucine the ratio leucine isoleucine is similar to that in animal proteins.

The total amino acid composition, free and bound, of the nitrogenous compounds of fraction I is close to that of the free amino acids of this fraction. The endogenous origin of the compounds is considered.

\section{RÉFÉRENCES BIBLIOGRAPHIQUES}

Ardis (h., Jolles P., rgot. Étude des substances solubles au cours de la réaction de la présure sur la caséine. Bull. Soc. Chim. Biol., 46, 7-8.

Borgstron B., Daillquist A., Gustafison B. li., Lundi G., Malmouist J., 1959. Trypsin, invertane, anylase content of fæces of Germ-frec Rats. J. Proc. Soc. Exper Biol. Med., 102, I54.

Combe E., pexot E., Chirtier H., Sacolet E., i965. Métabolisme du rat "germ-free". Ann. Biol. anim. Bioch. Biophys., 5, $189-206$.

Combe İ., SAçuer E., ig66. Influence de l'cetat axénique sur divers composés azotés contenus dans le cacum de rats albinos recevant des quantités variables de proténes. C. R. Acad. Sci. Ser. D., 262. 685 -68s.

Gordon II. A., 1959. Characterization of germ-free life. Inn. Y. Y. Acad. Sci., 78, 208-22o.

Lindstedt G., LindstedT S., Gustafison B.E., I965. Mucus in intestinal contents of Germ free rats. J. Exper. Med., г г 2, р. 201-2г3.

Werner I., 1953. Studies on glycoprotein from mucous epithelium aurl epithelial secretions. Acta Soc. .Med. Upsal., 58. 1-5.5. 